\title{
Multiple metastasis of follicular variant of papillary thyroid carcinoma coexistent with malignant melanoma
}

\author{
Yu Ji Kim, Kyung Ae Lee, Tae Sun Park, Hong Sun Baek, and Heung Yong Jin
}

Division of Endocrinology and Metabolism, Department of Internal Medicine and Research Institute of Clinical Medicine of Chonbuk National University-Chonbuk National University Hospital, Chonbuk National University Medical School, Jeonju, Korea
Received: September 13, 2015

Revised : January 18, 2016

Accepted: March 27, 2016

\section{Correspondence to}

Heung Yong Jin, M.D.

Division of Endocrinology and Metabolism, Department of Internal Medicine, Chonbuk National University Medical School, 20 Geonji-ro, Deokjin-gu, Jeonju 54907, Korea

Tel: $+82-63-250-2474$

Fax: +82-63-254-1609

E-mail: mdjinhy@jbnu.ac.kr
To the Editor, Papillary thyroid cancer (PTC) is the most common type of differentiated thyroid cancer. Many subtypes of PTC have been described, and follicular variant papillary thyroid cancer (FVPTC) is a common subtype that is found in $23 \%$ to $41 \%$ of patients with PTC [1]. FVPTC is defined by the presence of tumor cells arranged almost entirely in a follicular architectural pattern, with nuclear features identical to those of conventional PTC. It has also been hypothesized that FVPTC behaves clinically in a way that is similar to conventional PTC [1].

Malignant melanoma is a skin cancer that is caused by the malignant transformation of melanocytes. Several recent studies have reported an association between PTC and malignant melanoma based on a genetic link related to the high prevalence of a mutation in the BRAF oncogene [2]. Other studies have reported the presence of the BRAF mutation in FVPTC [3]. Therefore, there may be a relationship between FVPTC and malignant melanoma. Here, we have described a FVPTC with multiple metastases combined with a malignant melanoma that did not respond to conventional treatment.

A 68-year-old female patient presented to our endocrinology department for evaluation of a palpable mass in the anterior neck in December 2008. She had 10-year history of hypertension and no family history of thyroid disease. On physical examination, both lobes of the patient's thyroid were enlarged. Thyroid ultrasonography (US) showed a 3.6 $\times 2.2 \mathrm{~cm}$, solid, hypoechoic nodule in the right thyroid lobe and about $3.0 \times 1.8$ $\times 5 \mathrm{~cm}$ sized, ill-defined, isoechoic nodule with microcalcifications in the left thyroid lobe. A neck contrast-enhanced computed tomography (CT) scan revealed a huge, heterogeneous enhanced mass accompanied by small foci of calcification in the thyroid gland. There was no evidence of regional invasion or metastasis to the lymph nodes. Serum levels of free thyroxine and thyroid stimulating hormone (TSH) were within normal range, and the chest $\mathrm{X}$-ray was unremarkable. US-guided fine-needle aspiration (FNA) of the thyroid was performed. The results of FNA cytology from the right thyroid nodule were suggestive of nodular hyperplasia. FNA cytology of the left thyroid nodule revealed malignant PTC cells. The patient underwent total thyroidectomy and central lymph node dissection. On gross examination, the left thyroid mass was well-demarcated and partially surrounded by a capsule, and it measured $6.0 \mathrm{~cm}$ at the longest dimension. The specimen from the right thyroid 
showed a well-circumscribed mass measuring $3.5 \times 3.0$ $\mathrm{cm}$. The histopathology analysis confirmed a FVPTC in the left lobe of the thyroid (Fig. 1) and a Hurthle cell adenoma in the right thyroid. No extrathyroidal local extension was seen. There was no evidence of vascular invasion or regional lymph node metastasis ( $\mathrm{p}^{\mathrm{T}} \mathrm{T}_{3} \mathrm{NoMo}$ ).

After total thyroidectomy, the patient was treated with high-dose (150 $\mathrm{mCi}$ ) radioactive iodine (RAI) therapy. At that time, the stimulated thyroglobulin $(\mathrm{Tg})$ level was 0.1 $\mathrm{ng} / \mathrm{mL}$, the anti-Tg antibody level was $1.87 \mathrm{U} / \mathrm{L}$ (reference
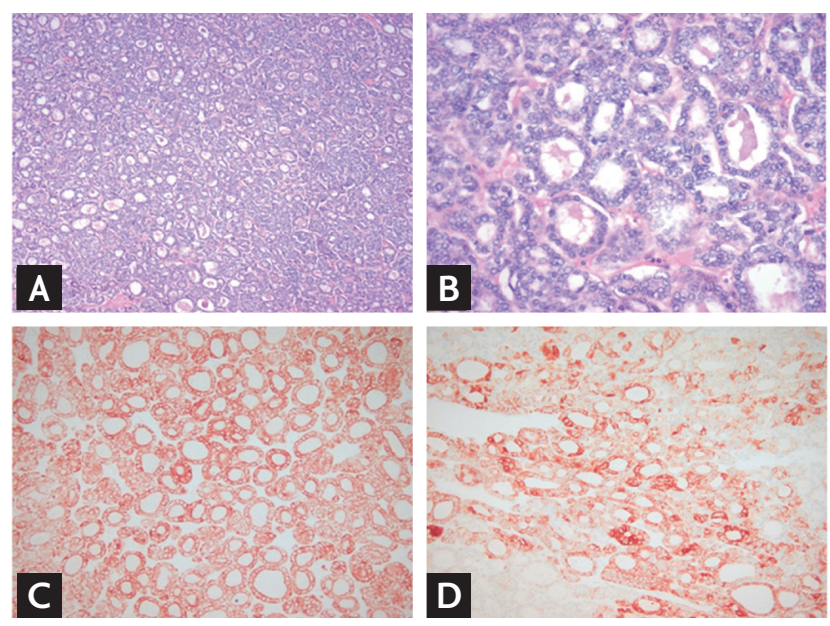

Figure 1. (A, B) Follicular variant papillary thyroid carcinoma. Follicles are of varying size and contain hyper-eosinophilic colloid. The nuclear morphology is clear and enlarged, with nuclear grooves (A: H\&E, ×100; B: H\&E, ×400). Follicular variant of papillary thyroid carcinoma is positive for $(C)$ CK 19 and (D) high-molecular weight cytokeratin (×200). range, o to 0.3 ), and the TSH level was $43.08 \mathrm{mIU} / \mathrm{L}$. A whole body scan (WBS) with I131 showed focal remnant uptake in the thyroid bed. Suppression therapy with 150 $\mu \mathrm{g}$ of levothyroxine was performed. Eight months later, she had no evidence of recurrence based on serum $\mathrm{Tg}$ levels (0.12 ng/mL) and neck US. However, she was non-compliant with levothyroxine and TSH level was often elevated. Furthermore, the patient refused follow-up stimulated Tg, neck US, and WBS.

Four years after the first RAI therapy, the patient's serum $\mathrm{Tg}$ level was elevated $(149.7 \mathrm{ng} / \mathrm{mL}$; normal range, o to 50), which was assumed to indicate recurrence of PTC. Positron emission tomography (PET)-CT was performed and revealed intense hypermetabolic lesions on the lung and sternum (Fig. 2A). There was no evidence of any other local tumor recurrence or distant metastasis except in the lung and sternum. These indicated a recurrence of PTC. She received $250 \mathrm{mCi}$ of second-round RAI therapy in October 2013. Despite RAI therapy, a follow-up iodine WBS still showed positive uptake in the sternum (Fig. 2B).

During TSH suppression therapy, the patient presented with a palpable nodule on the left flank in April 2014. There was a $3.5 \times 2.5 \mathrm{~cm}$ brown patch with $0.8-\mathrm{cm}$ central black nodule. A skin biopsy was performed and the histologic diagnosis was compatible with malignant melanoma. Subsequent PET-CT showed skin thickening in the posterolateral aspect of the left trunk with mild fluorodeoxyglucose uptake. There were no changes in
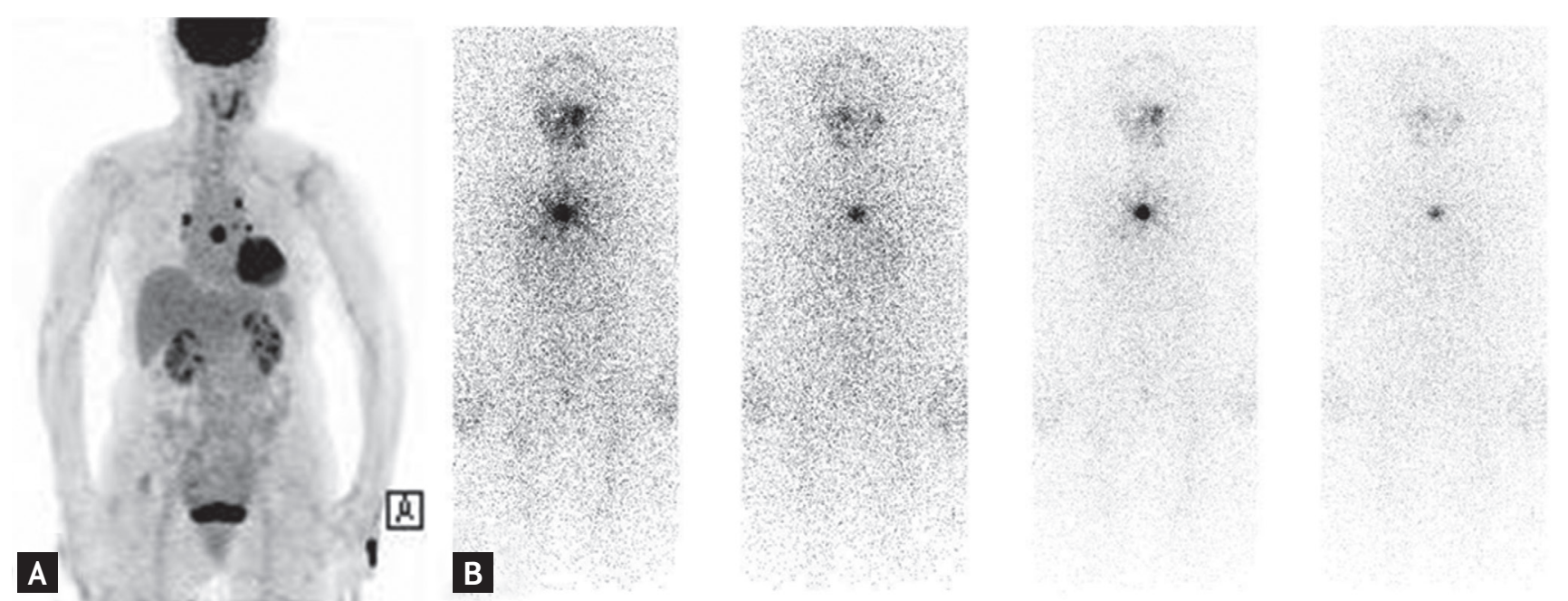

Figure 2. (A) Positron emission tomography-computed tomography revealed intense hypermetabolic lesions on the lung and sternum 4 years after total thyroidectomy. (B) Follow-up iodine whole body scan still showed positive uptake in the sternum after the 2 nd radioactive iodine therapy. 

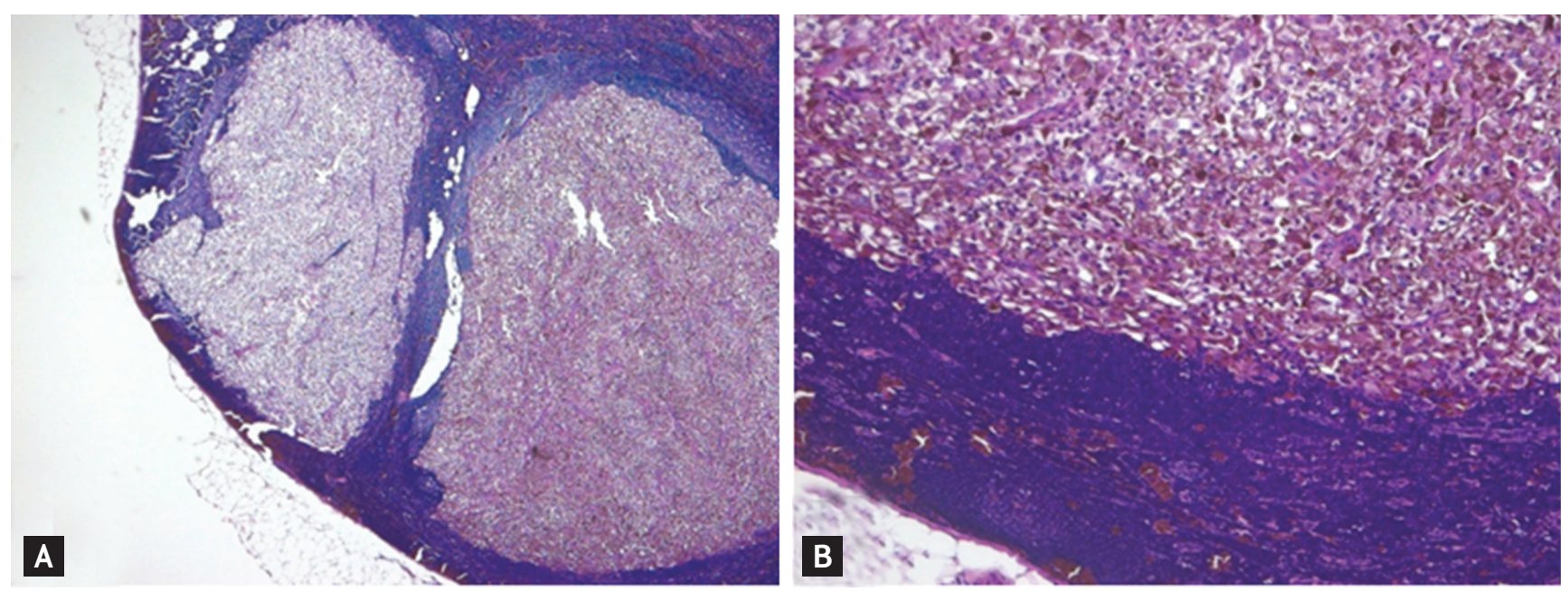

Figure 3. (A) Histologic findings showing a metastatic tumor component $(\mathrm{H} \& \mathrm{E}, \times 4 \mathrm{O})$.)(B) On high magnification, the tumor cells show nuclear atypia with prominent nucleoli and contain melanin pigment (H\&E, $\times 200)$.

the metastatic lesions in the lung and sternum, compared with the previous PET-CT image. She underwent surgical excision of the cutaneous malignant melanoma. On gross examination of the resected specimen, the pigmented lesion measured $2.8 \times 2.2 \mathrm{~cm}$ and was $1.5 \mathrm{~cm}$ away from the resection margin. The results of the histopathology analysis reported a Breslow depth of $2.4 \mathrm{~mm}$ (pT3a) and the patient was diagnosed with a superficial spreading melanoma with vertical phase growth pattern and without ulceration. Surgical margins were clear. A sentinel node biopsy was not performed.

After 4 months, the patient complained of new palpable axillary mass. US of the left axilla region showed an enlarged round lymph node with an irregular margin, without a fatty hilum. There was assumed to be a malignant lymph node. Resection of the metastatic lymph node was performed and pathologic findings were consistent with a metastatic malignant melanoma (Fig. 3). She started treatment with moderate-dose interferon therapy $\left(10 \mathrm{MU} / \mathrm{m}^{2}\right)$ in October 2014. The patient has continued moderate-dose interferon therapy and TSH suppression therapy. Additionally, a third round of highdose RAI therapy is planned for the remnant PTC lesion.

FVPTC is a major subtype of PTC. Tunca et al. [1] found that aggressive histopathologic features, such as thyroid capsule invasion, extrathyroidal extension, and lymph node metastasis, are significantly more frequent in conventional PTC than in FVPTC. In addition, this study found that long-term outcomes were comparable in both subtypes after appropriate initial surgery and postoperative RAI ablation treatment [1]. Molecular analysis shows that FVPTC has a BRAF mutation only $5 \%$ to $20 \%$ of the time, while conventional PTC shows a BRAF mutation more often (35\% to $70 \%$ ) [3].

Generally, malignant melanoma rarely occurs as a second primary tumor in patients with thyroid cancer. According to a previous report, a high frequency of BRAF mutation in patients with thyroid cancer and malignant melanoma was suggested to be the cause of the cooccurrence of the two tumors [2]. BRAF is thought to be the key RAF isoform transducing the cyclic adenosine monophosphate-dependent growth signal in both thyroid cells and melanocytes [4], which may explain the shared genetic vulnerability between PTC and malignant melanoma. As far as we know, co-occurrence of a specific histologic subtype of PTC with malignant melanoma has rarely been reported.

Other studies have reported that all lesions of melanocytic origin, benign or malignant, express TSH receptor, and that receptor expression is increased in melanomas, potentially rendering them more sensitive to TSH. Thus, TSH has been implicated in the malignant transformation of melanocytes to melanoma, and TSH plays a role as novel growth factor of human melanoma [5]. However, little is known about the exact genetic components or TSH levels that are involved the co-occurrence of PTC and malignant melanoma.

There are several uncommon aspects of this patient's 
presentation. First, the FVPTC was aggressive with distant metastases and coexisted with cutaneous malignant melanoma. Perhaps the relatively long period of TSH elevation when the patient was noncompliant promoted the aggressive nature of this FVPTC and the occurrence of malignant melanoma. Furthermore, a selective shared genetic alteration, such as a BRAF mutation, may increase the possibility that the two cancers may be connected through a common undiscovered pathogenic mechanism. Unfortunately, BRAF mutation analysis was not performed in resected specimens from the FVPTC and malignant melanoma. Second, this patient did not respond to treatment for the FVPTC metastasis or malignant melanoma. It remains unclear how the treatment of two coexisting tumors affects each tumor individually. It is possible that genetic alterations in cells from both tumors can affect therapeutic responsiveness.

This case emphasizes the need for awareness of the possibility that PTC and malignant melanoma can simultaneously occur; we recommend careful monitoring for malignant melanoma in PTC patients. Further studies are warranted to elucidate the genetic association between specific histologic subtypes of PTC and malignant melanoma and the response to treatment of two coexisting tumors.

Keywords: Follicular variant papillary thyroid carcinoma; Melanoma; Co-occurrence

\section{Conflict of interest}

No potential conflict of interest relevant to this article was reported.

\section{REFERENCES}

1. Tunca F, Sormaz IC, Iscan Y, Senyurek YG, Terzioglu T. Comparison of histopathological features and prognosis of classical and follicular variant papillary thyroid carcinoma. J Endocrinol Invest 2015;38:1327-1334.

2. Oakley GM, Curtin K, Layfield L, Jarboe E, Buchmann LO, Hunt JP. Increased melanoma risk in individuals with papillary thyroid carcinoma. JAMA Otolaryngol Head Neck Surg 2014;140:423-427.

3. Lloyd RV, Buehler D, Khanafshar E. Papillary thyroid carcinoma variants. Head Neck Pathol 2011;5:51-56.

4. Kimura ET, Nikiforova MN, Zhu Z, Knauf JA, Nikiforov YE, Fagin JA. High prevalence of BRAF mutations in thyroid cancer: genetic evidence for constitutive activation of the RET/PTC-RAS-BRAF signaling pathway in papillary thyroid carcinoma. Cancer Res 2003;63:14541457.

5. Ellerhorst JA, Sendi-Naderi A, Johnson MK, Cooke CP, Dang SM, Diwan AH. Human melanoma cells express functional receptors for thyroid-stimulating hormone. Endocr Relat Cancer 2006;13:1269-1277. 